\title{
XIV. The effect of gases on the contact difference of potential between metals at different temperatures
}

\section{O.W. Richardson F.R.S. \& F.S. Robertson M.I.E.E.}

To cite this article: O.W. Richardson F.R.S. \& F.S. Robertson M.I.E.E. (1922) XIV. The effect of gases on the contact difference of potential between metals at different temperatures, Philosophical Magazine Series 6, 43:253, 162-174, DOI: 10.1080/14786442208633859

To link to this article: http://dx.doi.org/10.1080/14786442208633859

曲 Published online: 08 Apr 2009.

Submit your article to this journal $₫$

Џll Article views: 3

Q View related articles $\longleftarrow$

Citing articles: 7 View citing articles 5 
XIV. The Effect of Gases on the Contact Difference of Potential between Metals at Tifferent Temperatures. By O. W. Richandson, F.R.S., Wheatstone Professor of Physics, and F. S. Robertson, M.I.E.E., Lecturer in Electrical Engineering, University of London, King's College*

THE present paper deals with some-observations of the curves connecting thermionic electron current and applied potential difference (characteristic curves) for the case of a straight hot wire and a coaxal metal cylinder when small known pressures of gases, principally hydrogen, are present. The currents dealt with were rather small, being of the order $7 \times 10^{-8} \mathrm{amp}$, and approximate saturation was attained under the experimental conditions with accelerating potentials of the order of a volt. Attention has been directed chiefly to the region in which the curves approach the voltage axis. In this region the shapes of the curves are determined by the distribution of velocity among the emitted electrons, by the geometry of the electrodes and the fall of potential down the hot wire. Whilst these facturs were not exactly identical in all the comparative experiments, it is believed that the effects of variation in them are practically negligible, with the result that the shapes of the different curves under comparison are invariable. This is true, as may be seen from the diagrams, figs. 2, 4, and 5, not only for the curves taken under high vacuum conditions, but also for those taken when small quantities of hydrogen were admitted.

Whilst in general the curves are of the same shape they are not in identical positions, but those obtained in the presence of hydrogen are displaced along the voltage axis as compared with those obtained in a vacuum. Inasmuch as for a given maximum or saturation current the shapes of the curves are the same, it is reasonable to infer that the infrasaturation currents will be sensibly the same function of the true voltage between the electrodes. Now the voltage plotted in the diagrams is the voltage between the central point of the hot wire and the surrounding cylinder as read on a voltmeter which in effect is inserted between these points. This differs from the true voltage by an amount equal to the contact difference of potential between the surfaces plus an effect arising from the drop of potential due to the heating current down the wire. With the arrangements used the last effect is believed to have been sensibly the same in all the experiments ; so that the displacement of the curves along the voltage axis should be, a measure of the change which took place in the contact potential difference between the hot

* Communicated by the Authors. 
wire and the surrounding cylinder under the conditions investigated.

Little need be said of the actual experimental arrangements, which were not for the most part of a novel character in thermionic work. For the production and maintenance of a high vacuum a mercury vapour pump backed by an oil box pump in series with a Geryck piston pump was used, and this could be supplemented when desirable or convenient by a liquid air cooled charcoal attachment. The tubes were mounted so that a vacuum furnace ${ }^{*}$ could be placed over them when desired, so as to get the gas out of the tube walls and electrodes. When desired pure hydrogen could be admitted by gently heating an attached palladium tube with a Bunsen burner and the pressure read off on a MeLeod mauge. The pressures of hydrogen used were all so small that they did not affect the shapes of the curves to any ascertainable extent by interference of the gas molecules with the motions of the electrons.

The simplest conditions with a view to the theoretical interpretation of the results would be obtained from the displacements of the curves for the emitting wire maintained at a constant temperature in the different experiments. This, however, involves a good many practical difficulties under the experimental conditions. The problem of maintaining a wire accurately at a constant temperature in presence of varying amounts of gas is one of great difficulty. In the case of platinum for example the effect of absorbed hydrogen on the resistance and the cooling effect of the hydrogen atmosphere vitiate the resistance method of holding the temperature constant. It is doubtful whether the methods of optical pyrometry as applied to thin filanents are sensitive enough for this purpose. Another difficulty arises from the very considerable effect of hydrogen in changing the emission constants of the metal. If a standard temperature is set up for some particular pressure, it may be that on changing to another pressure the emission will be so high that the shape of the characteristics is changed owing to the effect of the self-repulsion of the electrons or, on the other hand, it may be so low that the instruments available are not sensitive enough to make measurements of it. The last is hardly an insuperable objection as it may be overcome by changing over to more sensitive instruments ; but such changing over involves much time at the best, and rapidity of operation is probably the most important factor contributing to success in experiments of this kind.

* Richardson, 'Emission of Electricity from Hot Bodies,' p. 15. 


\section{Prof. Richardson and Mr. Robertson : Effect of}

Mainly for these reasons the procedure adopted in all cases has been, after each change in the conditions, so to regulate the temperature of the wire that the saturation current was maintained at approximately its original value. This could be done rapidly by means of a regulating rheostat. Whilst these conditions are not theoretically ideal they are at any rate pretty definite, and are probably the best that could be adopted for a fairly rapid preliminary examination of the phenomena. In many cases a sufficiently close idea of the actual temperature of the wire can be got from its dimensions and from knowledge already available as to the relation between emission and temperature under the given conditions. It is, however, important to remember that the comparisons made in this work are not between wires maintained at a given temperature with different gas pressures, but between wires at such various temperatures as will maintain the thermionic current constant. These various temperatures are in general a function both of the original high vacuum temperature and of the pressure of the admitted gas.

\section{Experiments with Platinum.}

The experiments with this metal were made with the tube shown in section in fig. 1 . The hot platinum wire was $3 \cdot 0 \mathrm{~cm}$. long and $0.184 \mathrm{~mm}$. diameter. The leads were of platinum $14.6 \mathrm{~cm}$. long and $0.52 \mathrm{~mm}$. diameter.

The cylinder was of piatinum foil and was $5.0 \mathrm{~cm}$. long and $1.88 \mathrm{~cm}$ diameter. The tube was cleaned by boiling nitric acid and distilled water before the experiments.

In the initial experiments the tube was only exhausted cold and with the wire glowing and was not baked out in the vacuum furnace. This was done so as to realise the conclitions referred to in the old experiments on the electron emission from platinum as those pertaining to a new wire*. Under these circumstances the characteristics were found to be fairly sensitive to small quantities of hydrogen. The admission of the hydrogen displaced the characteristics away from the direction of accelerating voltages, indicating that the hot wire had become relatively electronegative with

Fig. 1.

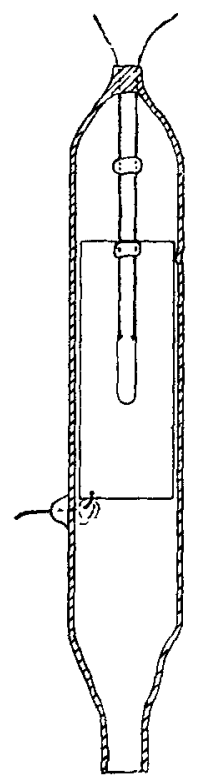

* Cf. 'Emission of Electricity from Hot Bodies,' Chap. iv. p. 102. 
Gases on Contact Potential Difference between Metals. 165

respect to the cylinder. Initially this displacement appeared to be quite reversible. Thus in a particular test starting with a pressure of $10^{-4} \mathrm{~mm}$. or less, the admission of 0.201 $\mathrm{mm}$. of hydrogen displaced the curve 35 volt to the left, on pumping the hydrogen out it moved back $\cdot 33$ volt (i. e. to within 0.02 volt of its original position). A typical set of curves obtained after a few hours heating is shown in fig. 2 .

\section{Fig. ?.}

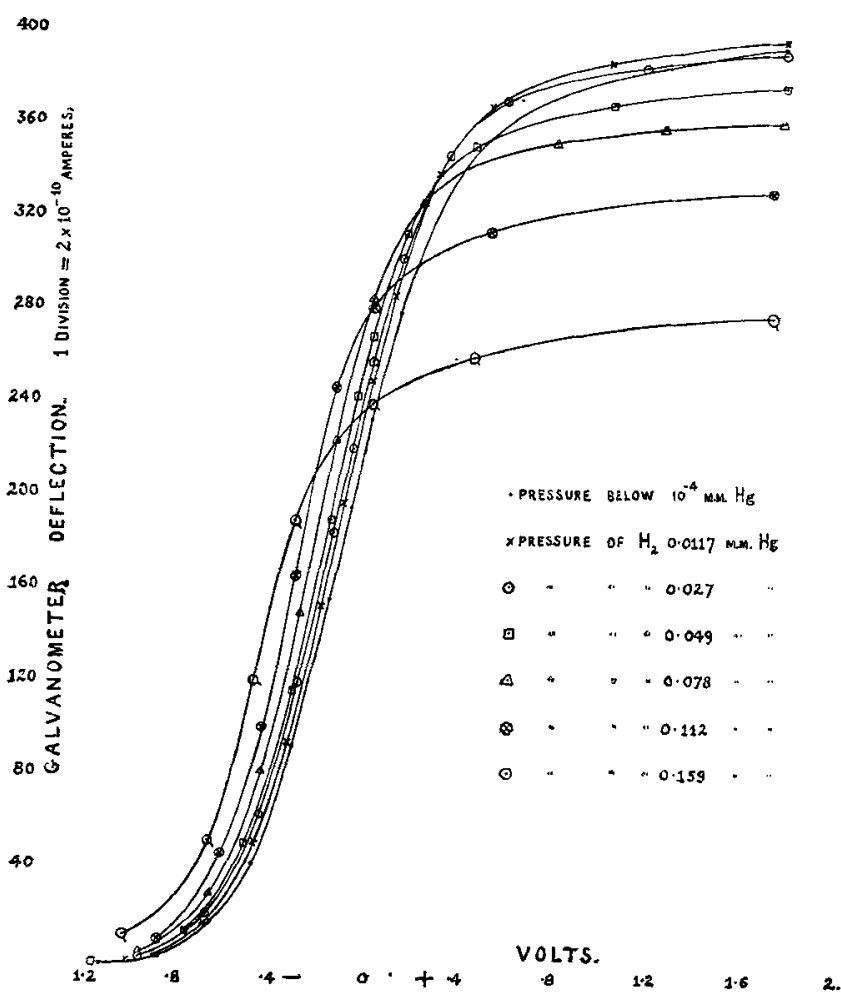

The temperature for the high vacuum curve in this case was about $1460^{\circ} \mathrm{K}$. The gas pressures at which the various curves were taken are marked on the figure. The displacements are very nearly proportional to the pressure of the admitted hydrogen, as is shown in the plot fig. 3 .

After heating for some time the curves became somewhat 
166 Prof. Richardson and Mr. Robertson : Effect of

less sensitive to the admission of hydrogen, and this condition persisted after the tube had been baked out in the furnace and re-exhausted. Typical eurves at this stage under clifferent pressures are shown in figs. 4 and 5. . Although the

Fig. 3.

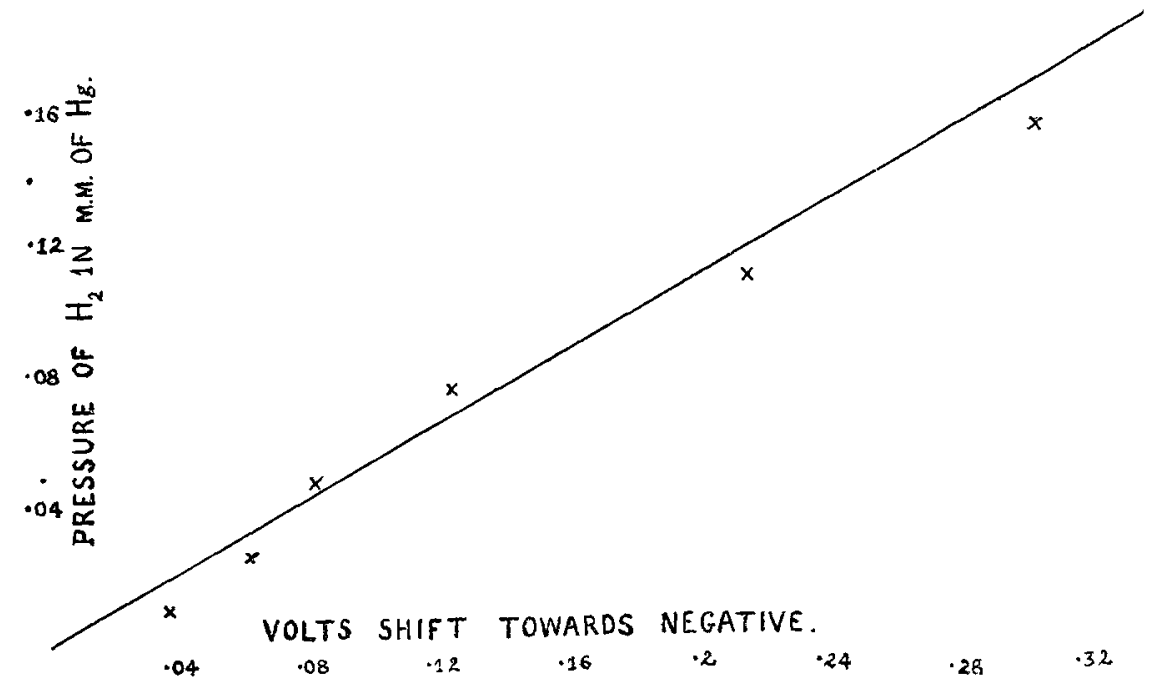

displacements are small a careful examination of all the data strongly suggests that they are real. The position of the point on the voltage axis for which the current had half the saturation value is given for a series of pressures in the following table:-

$\begin{array}{cccccc}\text { Pressure }\left(\mathrm{mm} . \times 10^{1}\right) \ldots \ldots & \ngtr 1 & 17 & 65 & 463 & 1000 \\ \text { Voltage for half value..... } & -.25 & -.30 & -.35 & -.41 & -.45\end{array}$

It will be seen that the voltage displacements for a given increment in pressure are greater when the pressure is low. This condition, of which figs. 4 and 5 are typical examples, appeared to be stable and persisted to the end of the experiments, in the course of which the wire was heated some. hundreds of hours. The displacements on reducing the pressure were generally somewhat smaller than those obtained when the pressure was increased between the same limits. 
Gases on Contact Potential Difference between Metals. 167

This is probably due to the time required to attain equilibrium between the gas and the metal.

Fig. 4.

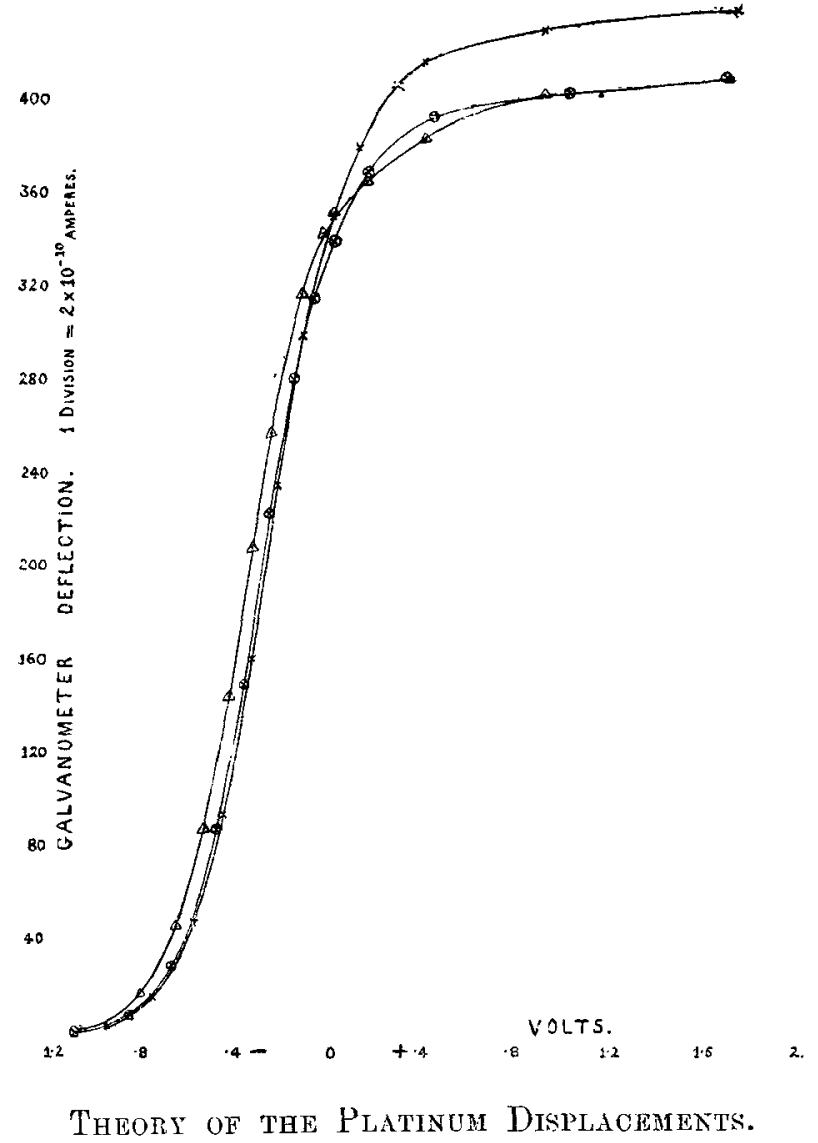

Whilst a complete test of the theory of these effects will require a more elaborate experimental investigation than the present, the results are in some respects definite enough to merit a theoretical discussion on broad lines. ' A theory of the connexion between contact electromotive force and electron emission from contaminated surfaces has been developed by one of the authors*, and is discussed in connexion with H. A. Wilson's theories of the effect of hydrogen on the

* Richardson, Roy. Soc. Pruc. A, vol. xci. p. 524 (1915). 
168 Prof. Richardson and Mr. Robertson : Etfect of

emission from platinum in the "Emission of Electricity from Hot Bodies,' Chap. iv. pp. 108 et seq., to which the following

Fig. 5.

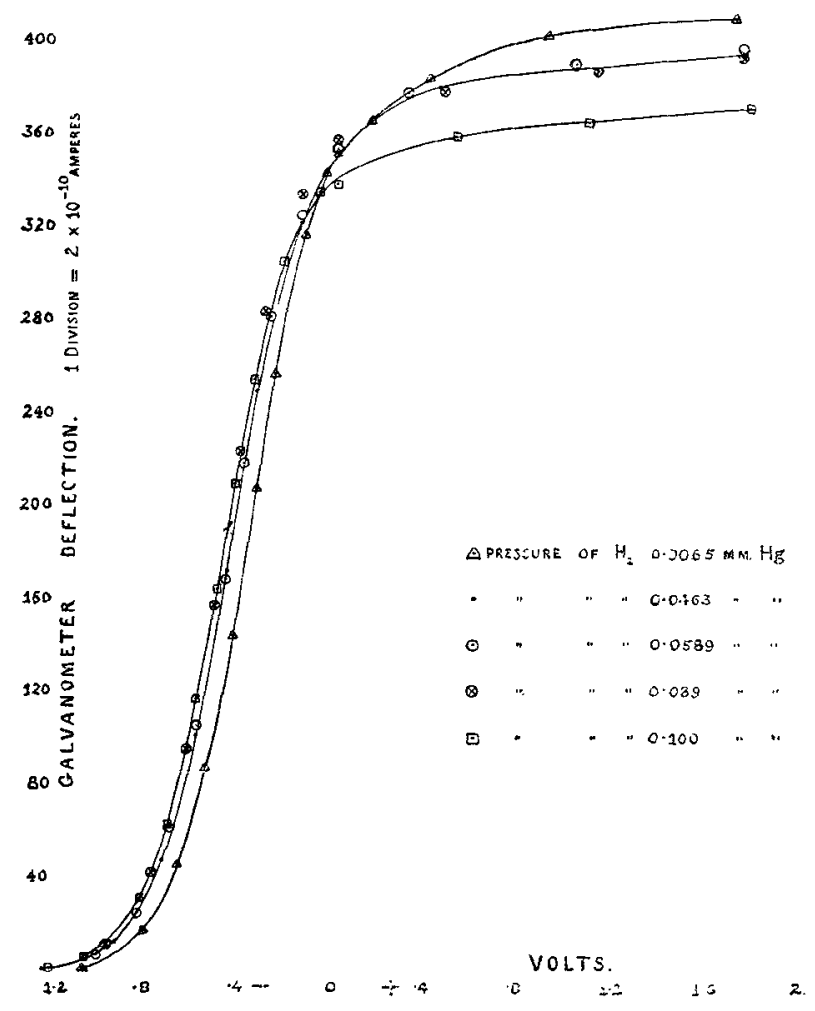

references relate. In general (equation $3 t^{\prime}$ p. 109 ), the contact potential difference between ary two surfaces at temperature $\mathrm{T}_{1}$ is

$$
\mathrm{V}_{1}=\frac{k \mathrm{~T}_{1}}{e} \log \frac{n}{n^{\prime}}=\frac{k \mathrm{~T}_{1}}{e} \log \frac{i}{i^{\prime}}, \quad . \quad . \quad .
$$

where $i$ and $i^{\prime}$ are the saturation currents per unit area of the two surfaces at $\mathrm{T}_{1}$. If the dashed variables refer to the clean metal and the undashed to the metal in presence of gas, it follows from (13) p. 112 that

whence

$$
i \mid i^{l}=\left(1+a p^{c}\right)^{\left(\frac{\alpha}{c}-\frac{1}{\mathrm{~T}_{1}}-1\right)}, \quad \text {. . . . . }
$$

$$
\mathrm{V}_{\mathrm{I}}=\frac{k \mathrm{~T}_{1}}{e}\left(\frac{\alpha}{e^{\mathrm{T}} \mathrm{T}_{1}}-1\right) \log \left(1+\alpha,^{c}\right) . \quad . \quad .
$$


Gases on Contact Potential Difference between Metals. 169

For platinum when it is sensitive to hydrogen the constants $a, c$, and $\alpha$ have the values $a=1.27 \times 10^{4}, c=0.73$, and $\alpha=2 \cdot 43 \times 10^{3}$ when $p$ is in millimetres of mercury.

The contact potential which is operative during the experiments is not $V_{1}$, which refers to a gap between two metal surfaces both at temperature $T_{1}$ when one is in a gas-free space and the other in hydrogen at pressure $p$, but it is the potential difference between two surfaces at temperatures $\mathrm{T}_{1}$ (that of the hot wire) and $\mathrm{T}_{0}$ (that of the cold cylinder), both in an atmosphere of hydrogen at pressure $p$. Let us call this potential difference $\Omega$. To find the value of $\Omega$ consider the gap between two blocks of the metal, one maintined at temperature $\mathrm{T}_{1}$ and the other at $\mathrm{T}_{0}$, both inmersed in hydrogen at pressure $p$ and connected together by a platinum wire. Next consider the work done in taking an electron round a closed circuit starting inside the metal at $\mathrm{T}_{1}$, across the boundary of $\mathrm{T}_{1}$, across the gap, then across the boundary of $\mathrm{T}_{0}$, and finally down the wire to the starting point. 'This consists of the algebraic sums of the amounts of work done in crossing five surfaces, viz. that between the pure and contaminated metal at $\mathrm{T}_{1}\left(\eta_{1}\right)$, that between the contaminated metal at $\mathrm{T}_{1}$ and the gas $\left(\phi_{1}\right)$, that across the gap $(-e \Omega)$, that from the gas to the contaminated metal at $\mathrm{T}_{0}\left(\phi_{0}\right)$, and thit between the pure and contaminated metal at $\mathrm{T}_{0}\left(\eta_{0}\right)$. Thus by the energy principle

$$
e \Omega=\phi_{1}+\eta_{1}-\left(\phi_{0}+\eta_{0}\right)
$$

The notation is the same as that in 'Emission of Electricity etc.' p. 109. In the same notation (p. 109 equation $34^{\prime}$ ) we have

and similarly

$$
e \mathrm{~V}_{1}=\phi_{1}^{\prime}+\eta_{1}^{\prime}-\left(\phi_{1}+\eta_{1}\right), \quad \cdot \quad \cdot \quad \cdot
$$$$
e V_{0}=\phi_{0}{ }^{\prime}+\eta_{0}{ }^{\prime}-\left(\phi_{0}+\eta_{0}\right) . \quad \text {. . . . }
$$

Now $\eta_{1}^{\prime}=\eta_{0}^{\prime}=0$; so that

$$
e \Omega=e\left(V_{0}-V_{1}\right)+\phi_{1}{ }^{\prime}-\phi_{0}{ }^{\prime} . \quad . \quad . \quad .
$$

Now, from loc. cit. p. 33 equation (16) (equation (15) p. 32 is more accurate but the difference is hardly material)

$$
\phi_{1}{ }^{\prime}-\phi_{0}{ }^{\prime}=\frac{3}{2} k\left(\mathrm{~T}_{1}-\mathrm{T}_{0}\right), \quad \text {. . . . }
$$

and substituting for $V_{0}$ and $V_{1}$ from (3) above and the similar equation for $\mathrm{V}_{0}$

$$
\Omega=\frac{k}{e}\left(\mathrm{~T}_{1}-\mathrm{T}_{0}\right)\left\{\frac{3}{2}+\log \left(1+u p^{c}\right)\right\}, . .
$$

or, a little more accurately,

$$
\Omega=\frac{k}{e}\left(\mathrm{~T}_{1}-\mathrm{T}_{0}\right)\left\{\frac{3}{2}+\log \left(1+a p^{c}\right)\right\}-\int_{1_{0}}^{\mathrm{T}_{1}} \sigma d \mathrm{~T} .
$$


Now we have seen (p. 164, ante) that $\mathrm{T}_{1}$ valried in the different comparative experiments, being a function of the temperature $\mathrm{I}_{2}$ of the hot wire in the high vacum experiments and the pressure $p$ of the gas. It was in fact the temperature which at the pressure $l$ gave the emission its high vacuum value and is therefore determined by the equation

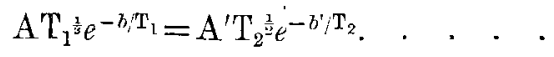

In this equation the factors $\mathrm{T}_{1}^{\frac{1}{2}}$ and $\mathrm{T}_{2}^{\frac{3}{2}}$ can be treated with sufficient exactness as equal; so that to such approximation as we require

$$
\mathrm{T}_{1}=\frac{b}{b}+\mathrm{T}_{2} \log \mathrm{A} / \mathrm{A}^{\prime}, \mathrm{T}_{2}, \quad . \quad . \quad . \quad .
$$

and using equations equivalent to loc.cit. p. 112 (15) and (17),

$$
\mathrm{T}_{1}=\frac{b^{\prime}-\frac{\alpha}{c} \log \left(1+a p^{c}\right)}{b^{i}-\frac{c}{\mathrm{~T}_{2}} \log \left(\overline{1+a p^{c}}\right)} \mathrm{T}_{2}, \quad . \quad . \quad . .
$$

and the operative contact potential in volts is

$$
\begin{aligned}
\frac{\mathrm{V}}{300}=\left\{\frac{b^{\prime}-\frac{\alpha}{c} \log \left(1+a p^{c}\right)}{b^{\prime}-\mathrm{T}_{2} \log \left(1+a p^{c}\right)} \mathrm{T}_{2}-\mathrm{T}_{0}\right\} \\
\quad \times\left\{\frac{k}{e}\left(\frac{3}{2}+\log \left(1+a p^{c}\right)\right)\right\}-\int_{\mathrm{T}_{0}}^{\mathrm{T}_{1}} \sigma d \mathrm{~T} .
\end{aligned}
$$

Equations (21) and (22) express $V$ as a function of the temperature $\mathrm{T}_{2}$ of the hot wire in the high vacrum experiments, $\mathrm{T}_{0}$ that of the cold electrode, the pressure $p$ of the hydrogen, the universal constants $k$ and $e$, the constants $\alpha$, and $a$ defined already, the emission constant $b^{\prime}$ for the metal in a vacuum, and the specific heat of electricity $\sigma$ in platinum. The term in $\sigma$ is unimportant, but is added for the sake of completeness.

If this theory is correct the observed displacements of the curves will be equal to the differences in the values of $V$, for a given value of $\mathrm{T}_{2}$, corresponding to the gas pressures used. Neglecting the unimportant term in $\sigma$ and substituting the known values of the constants, the values of $V$ given by equation (22) are :-

For $\mathrm{T}_{2}=1200^{\circ} \mathrm{K}$ : when $p=0.112 \mathrm{~mm}$. $\mathrm{V}=0.4 .52$ volt, and when $p=0.0013 \mathrm{~mm}$. $\mathrm{V}=0.374$ rolt. 
The displacement for this limit of pressure would thus be $\delta \mathrm{V}=\cdot 078$ volt .

For $\mathrm{T}_{2}=1460^{\circ} \mathrm{K}$ : when $p=0.112 \mathrm{~mm} . \mathrm{V}=0.629$ volt, and when $p=0 \cdot 0013 \mathrm{~mm}$. V $=0 \cdot 504$ volt.

For this value of $\mathrm{T}_{2}, \delta \mathrm{V}=0.125$ volt.

The experimental value of the displacement over this range of pressure for $\mathrm{T}_{2}=1460^{\circ} \mathrm{K}$. was $0 \cdot 16$ volt ; so that the observed effect is in fair accordance with the theory. It should also be added that it is in the prescribed direction.

There is one point in this discussion which perhaps has not received sufficient emphasis, namely, that it appears vitsl in order to reconcile the results with the type of theory dealt with to assume that the hydrogen is in equilibrium not merely with the surface of the hot platinum wire but with that of the cold platinum foil as well. If the hydrogen did not modify the cold platinum surface this would act as a reference surface of constant potential, and a calculation along similar lines for this case shows that the effects should be considerably greater than those observed and in the opposite direction. This is in accordance with the reguirement that the hydrogenated platinum surface should be electropositive to a clean platinum surface at all temperatures. The physical reason for the opposite direction of the effect as given by the experiments and supported by the calculations, lies in the fact that the temperature coefficient of the electron work function is negative at all pressures and increases with the pressure of the hydrogen.

\section{Experiments with Tungsten.}

We have made a large number of experiments with tungsten filaments using the type of tube shown in fig. 6 . The anodes were of copper foil and were $5.0 \mathrm{~cm}$. long and $1.9 \mathrm{~cm}$. in diameter. The tungsten filaments were $3 \cdot 0$ $\mathrm{cm}$. long and $0.127 \mathrm{~mm}$. in diameter except in the data shown in fig. 7 where the diameter was $0.076 \mathrm{~mm}$. The saturation currents were of the same order as before, $10^{-7} \mathrm{amp}$. The wires were kept taut along the axis of the cylinder by means of the molybdenum wire springs shown at the ends. These were under slight tension and took up the slack due to expansion on heating.

For some reason we have found it more difficult to get consistent results with tungsten than with platinum. This may be due partly to inexperience in controlling the conditions as the tungsten experiments were, in point of fact, 
172 Prof. Richardson and Mr. Robertson : Liffect of

made first. It is probable, however, that the main factor in this is the superior chemical activity of tungsten and copper as compared with platinum. Anotber difficulty arises from the very marked "clean up" effects with tungsten, which for example when working with hydrogen cause the pressure of the gas to vary considerably in a single experiment. It would take too much space to discuss the whole mass of data which have been obtained in cletail, and we shall merely point out the more important conclusions which follow from the experiments.

The first point tested was as to whether this admission of small amounts of mereury vapour at a pressure of the order $\cdot 001 \mathrm{~mm}$. would displace the curves along the voltage axis. No displacement was observable, indicating that mercury vapour does rot affect the contact potential between hot tungsten and cold copper. This is in agreement with the observation * that the electron emission from tungsten is not affected by mercury vapour. In these experiments the mercury vapour was only admitted for short intervals. No doubt if enough were admitted to amalgamate the surface of the cold electrode some displacements would be anticipated.

The problem of the effect of hydrogen was attacked in a similar way to that in Fig. 6.

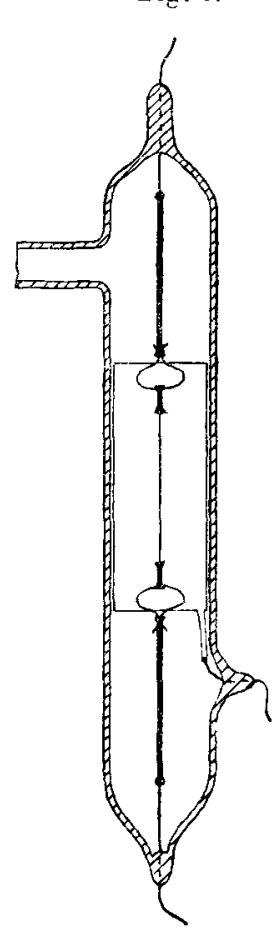
the case of platinum. The experiments show that any genuine effect of small quantities of hydrogen is small, and we have not been able to convince ourselves that there is such an effect. The most complete set of data gave the following values for the potentials $V$ on the roltage axis at which the currents had attained half the saturation value at the pressures stated :-

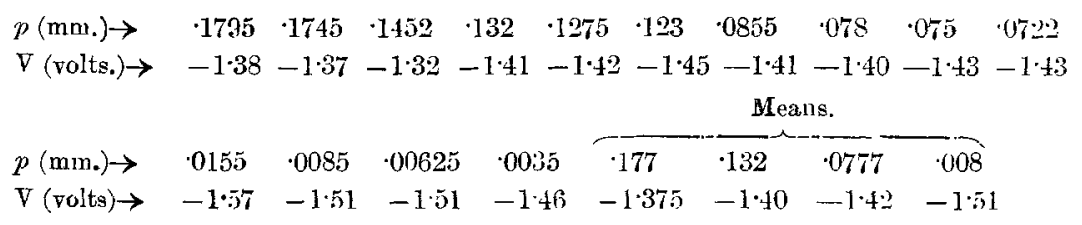

* lichardsoll, Phil. Mag. vol. xxvi. p. $34 t$ (1913). 
Gases on Contact Potential Difference between Metals. 173

These figures suggest that if the effect is real it is in the opposite direction to that observed with platinum, i.e. the hydrogen tends to make the hot tungsten more electropositive with reference to the cold electrode. However, further tests were inconclusive, the gas sometimes apparently causing a small displacement in one direction and at other times in another. All that we are prepared to state definitely at present is that there is no effect of an order of magnitude exceeding that indicated by the figures given above.

Fig. 7.

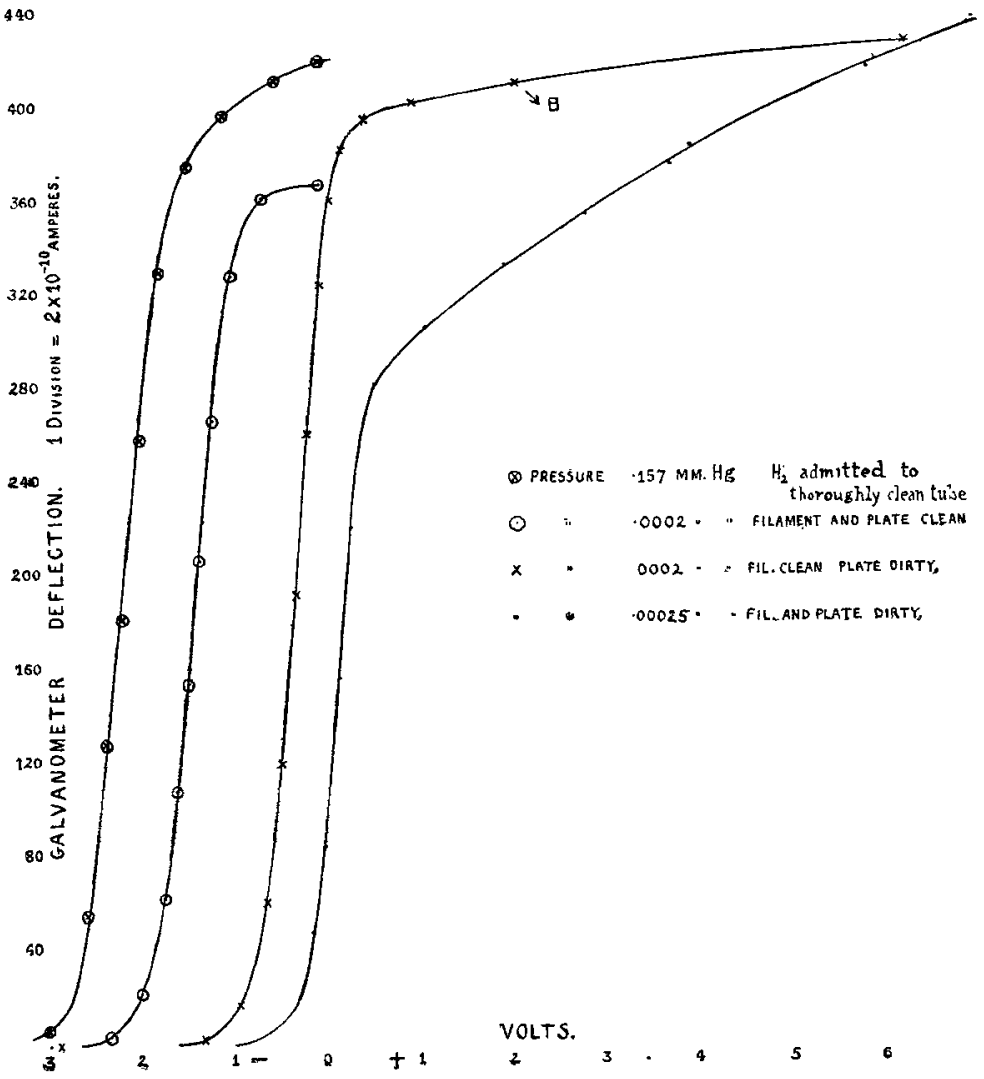

From time to time, and generally after the apparatus had undergone some drastic treatment, displacements in the characteristics of an entirely different order of magnitude were observed. It seems fairly certain that these large 


\section{Prof. Eddingten : Significance of Einstein's Gravitational}

effects are due to contaminations, chiefly by oxidation, of the surfaces of the tungsten filament and the copper anode. The extreme variations which have been observed for the position of the voltage at which the current attained half the saturation value are -2.11 and +0.17 ; the corresponding curves are shown in fig. 7. This corresponds to a total change in the contact potential difference of $2 \cdot 28$ volts. The extreme displacements in the positive direction along the voltage axis, such as that with the half saturation value at $+\cdot 17$ in fig. 7 , were obtained when the tube had been well baked out and the tungsten filament had been intentionally oxidized by glowing in air at a low pressure with the tube cold before testing. The evidence seems clear that an oxidized filament is electropositive by about 0.6 volt as compared with a clean filament at these temperatures.

When the filaments were oxidized the currents were found to be difficalt to saturate. This is shown by the upward slope of the relatively flat part of the curve on the right in tig. 7. There seems to be no doubt that this upward slope is due to the oxidized conditions of the tungsten surface. On glowing out the tungsten at a high temperature so as to cause the layer of oxide to evaporate, curves such as B fig. 7 were obtained. These are displaced about 0.6 volt to the left, corresponding to the more electronegative character of the clean tungsten, and at the same time the upward slope of the saturation part is found gradually to disappear.

$\mathrm{XV}$. On the significance of Einstein's Gravitational Equations in terms of the Curvature of the World. By A. S. Edington, M.A., F.R.S., Plumian Professor of Astronomy in the University of Cambridge*.

T $\mathrm{N}$ the Appendix to the paper on "The Relativity of Field and Matter" $\dagger, I$ gave a proof of the theorem that Einstein's equations $G_{\mu \nu}=\lambda g_{\mu \nu}$ express the condition that the radius of curvature of sections of the world is the same at all points and for all directions. I have since found that this proof is not sufficiently general. I took too limited a view of the vagaries of which a four-dimensional surface is capable when it has six extra dimensions to twist about in. I assumed that, although ten dimensions of Euclidean space are necessary for the representation of a four-dimensional Riemannian space, a small portion of the latter near the

* Coumunicated by the Author.

+ Phil. Mag. November 1921, p. 800. 